\title{
Clinical Utility of Trabecular Bone Score (TBS) in Fracture Risk Assessment of Patients with Rheumatic Diseases Treated with Glucocorticoids
}

\author{
Authors

\begin{abstract}
Affiliations
1 Department of Rheumatology, National Institute of Geriatrics, Rheumatology, and Rehabilitation, Warsaw, Poland

2 Department of Radiology, National Institute of Geriatrics, Rheumatology, and Rehabilitation, Warsaw, Poland
\end{abstract}

Anna Nowakowska-Płaza', Jakub Wroński' ${ }^{10}$, Iwona Sudoł-Szopińska², Piotr Głuszko
Key words

osteoporosis, glucocorticoids, fractures, trabecular bone score

received 24.01.2021

accepted after revision $\quad 10.06 .2021$

Bibliography

Horm Metab Res 2021; 53: 499-503

DOI 10.1055/a-1528-7261

ISSN $\quad 0018-5043$

(c) 2021. Thieme. All rights reserved.

Georg Thieme Verlag, Rüdigerstraße 14,

70469 Stuttgart, Germany

Correspondence

Jakub Wroński

Spartanska 1

02-637 Warsaw

Poland

Tel.: + 48 226709213, Fax: +48226709327

jakub-wronski@wp.pl
Supplementary material for this article is available under https://doi.org/10.1055/a-1528-7261

\section{ABSTRACT}

Chronic glucocorticoid therapy is associated with osteoporosis and can cause fractures in up to $50 \%$ of patients. Increased risk of fractures in patients with glucocorticoid-induced osteoporosis does not result only from the decreased bone mineral density (BMD) but also bone microarchitecture deterioration. Trabecular bone score (TBS) is a method complementary to DXA, providing additional information about trabecular bone structure. The aim of this study was to assess the clinical utility of TBS in fracture risk assessment of patients treated with glucocorticoids. Patients with rheumatic diseases treated with glucocorticoids for at least 3 months were enrolled. All recruited patients underwent DXA with additional TBS assessment. We analyzed the frequency of osteoporosis and osteoporotic fractures and assessed factors that might be associated with the risk of osteoporotic fractures. A total of 64 patients were enrolled. TBS and TBS T-score values were significantly lower in patients with osteoporosis compared to patients without osteoporosis. Low energy fractures occurred in 19 patients. The disturbed bone microarchitecture was found in $30 \%$ of patients with fractures without osteoporosis diagnosis based on BMD. In the multivariate analysis, only TBS and age were significantly associated with the occurrence of osteoporotic fractures. TBS reflects the influence of glucocorticoid therapy on bone quality better than DXA measured BMD and provides an added value to DXA in identifying the group of patients particularly prone to fractures.

\section{Introduction}

Treatment with glucocorticoids (GCs) is associated with a variety of adverse effects including osteoporosis, which can occur in up to $50 \%$ of patients with chronic GCs therapy [1,2]. The fractures associated with osteoporosis result in disability, impaired quality of life, increased mortality, and are a tremendous burden, both personal and economic [3]. The fracture risk in people with glucocorticoid-induced osteoporosis $(\mathrm{GIO})$ increases with the duration of therapy and doses of GCs. The risk is increased even in patients using GCs in as small doses as $2.5 \mathrm{mg}$ of prednisolone per day and with GCs therapy longer than 3 months [4]. Dual X-ray absorpti- ometry (DXA) of the proximal femur (hip) and lumbar spine, measuring bone mineral density (BMD), is the gold standard technique for osteoporosis diagnosis. However, the increased risk of low-energy fractures in patients with GIO does not result only from decreased BMD but also from bone microarchitecture deterioration and myopathy $[1,2,4]$. DXA BMD measurement does not assess the quality of trabecular bone structure, and therefore may not detect all patients at risk of low-energy fractures. To better assess the risk of fractures, tools that would allow an easy assessment of bone microarchitecture were sought. 
Currently, the most widely used tool for the evaluation of bone microarchitecture is the Trabecular Bone Score (TBS). TBS iNsight is a software tool available for DXA scanners, that enables the assessment of bone texture (an index correlated to bone microarchitecture) based on gray-level variations in DXA scans of the lumbar spine. The result is expressed as TBS, with higher scores in patients with better quality bone microarchitecture. TBS is a method complementary to DXA, providing additional information about the bone structure, and is an independent predictor of fracture risk [5]. European guidance for the diagnosis and management of osteoporosis in postmenopausal women emphasizes that TBS can supplement the FRAX calculator in the estimation 10-year risk of fractures [6].

TBS may be particularly useful in some types of secondary osteoporosis, where bone quality is especially affected [7]. Several studies have shown the usefulness of TBS in assessing bone microarchitecture in $\mathrm{GIO}$ [8], osteoporosis associated with diabetes mellitus [9], primary hyperparathyroidism [10], and chronic kidney disease[11]. More importantly, TBS seems to be more sensitive than BMD for the detection of fracture risk related to GCs therapy [12]. The aim of this study was to assess the clinical utility of TBS in fracture risk assessment in patients with inflammatory rheumatic diseases treated with GCs. So far, this issue has only been addressed in a few studies [13-16]. To our knowledge, this is also the first study performed in the Polish population.

\section{Subjects and Methods}

\section{Patients}

The study was conducted at the Department of Radiology in the National Institute of Geriatrics, Rheumatology, and Rehabilitation in Warsaw, Poland. Patients treated with GCs (prednisone dose $\geq 5 \mathrm{mg}$ per day or other glucocorticoids with equivalent dose) for at least 3 months were enrolled. The exclusion criteria were: age $<20$ years, BMI $<17 \mathrm{~kg} / \mathrm{m}^{2}$ or $>37 \mathrm{~kg} / \mathrm{m}^{2}$ (criteria provided by the manufacturer [17]), patients with diabetes mellitus or primary hyperparathyroidism, and patients with significant motor impairment preventing proper DXA examination. All recruited patients underwent DXA with additional TBS assessment. The study protocol has been approved by the hospital bioethics committee (KBT2/3/2019). All participants have signed informed consent for inclusion in the study. The study was conducted according to the Declaration of Helsinki.

\section{Measurements}

DXA scans were performed using Hologic Discovery A densitometer. DXA reports included the BMD value, expressed as grams per square centimeter $\left(\mathrm{g} / \mathrm{cm}^{2}\right)$, the T-score, and the Z-score values. In all analyzes of femur DXA reports the lower BMD value of the femoral neck or the total hip was used. As recommended by WHO, the T-score and Z-score were evaluated in analyzes depending on patient age. T-score was taken into account for postmenopausal women and men over 50, while Z-score for premenopausal women and men under 50 . According to the WHO definitions, in men over 50 and postmenopausal women osteoporosis was diagnosed when
T-score was $\leq-2.5$, and osteopenia when T-score was $<-1$ and $>-2.5$ [18]. In men under 50 years of age and women before menopause, osteoporosis was diagnosed based on Z-score $\leq-2.0$. Osteoporosis was also diagnosed in patients with osteopenia with low-energy fractures according to current guidelines [6].

TBS was assessed by automated analysis of lumbar spine DXA results using TBS iNsightversion 3.0.3.0 software. The TBS reports included the absolute TBS value and the TBS-T score for the sum of $\mathrm{L} 1-\mathrm{L} 4$ vertebrae. The absolute values of TBS were divided into those suggesting disturbed bone microarchitecture $(<1.23)$, intermediate $(\geq 1.23$ and $<1.31)$, or normal $(\geq 1.31)$ [19].

\section{Statistical analysis}

We analyzed the frequency of osteoporosis and osteoporotic fractures (depending on demographic and clinical characteristics or DXA/TBS results), the correlation between TBS and classical DXA parameters, the correlation of DXA/TBS parameters with patients' characteristics, and assessed factors that might be associated with the risk of osteoporotic fractures. The compliance of the data with the normal distribution was assessed using the Shapiro-Wilk test. The significance of the observed differences between the two groups was assessed using the Student's $t$-test for variables with a normal distribution, the Mann-Whitney U-test for variables without a normal distribution, and for categorical variables the Chisquare test or the Fisher's exact test (for tables with values < 5). For more than two groups with normal distribution, we used analysis of variance with posthoc analysis with Bonferroni test. For more than two groups without normal distribution, we used KruskalWallis test and Dunn's test respectively. The correlation was assessed using the Pearson correlation coefficient for parametric variables and Spearman's rank correlation coefficient with non-parametric variables. The significance of the correlation after adjusting for the confounding factors was checked by linear regression. To identify the predictors of osteoporotic fractures, logistic regression was used, with the backward stepwise analysis. Statistical significance was set at $p<0.05$. Statistical analysis was performed using Statistica 13.1 software.

\section{Results}

A total of 64 patients ( 49 females and 15 males) were enrolled. The patient characteristics are presented in $>$ Table 1. All patients were treated with GCs due to inflammatory rheumatic diseases - the majority suffered from rheumatoid arthritis ( $53.1 \%$ of all patients). Osteoporosis was diagnosed in 27 patients (42.2\%) - in 17 patients based on DXA, and in 10 patients based on the occurrence of low-energy fractures. Osteoporotic fractures (mostly vertebral fractures $63 \%$ ) occurred in 19 patients. According to TBS, 26 patients (40.6\%) revealed disturbed bone microarchitecture (TBS <1.23).

The TBS and TBS T-score values were significantly positively correlated with other bone density parameters - lumbar BMD, lumbar T-score, femoral BMD, femoral T-score ( $\triangleright$ Table 1S). The correlation of patients characteristics with the parameters of bone density and microarchitecture was assessed ( $\bullet$ Table 2 ) - showing a negative correlation of BMI with TBS values (TBS and T-score TBS), as well as a negative correlation of age with some BMD results (lumbar BMD, femoral BMD, and femoral T-score). Female gender was 
- Table 1 Patients characteristics.

\begin{tabular}{|l|r|}
\hline Sex, number (\%) & $49(76.6 \%)$ \\
\hline female & $63(20.87)$ \\
\hline Age, median (min, max) & $27.4( \pm 4.9)$ \\
\hline BMI, mean ( \pm SD) & $54(3,480)$ \\
\hline GCs treatment duration, median in months (min, max) & \\
\hline Disease, number (\%) & $34(53.1 \%)$ \\
\hline Rheumatoid arthritis & $9(14 \%)$ \\
\hline Systemic lupus erythematosus & $4(6.3 \%)$ \\
\hline Giant cell arteritis & $4(6.3 \%)$ \\
\hline Polymyalgia rheumatica & $4(6.3 \%)$ \\
\hline Vasculitis & $3(4.7 \%)$ \\
\hline Systemic sclerosis & $6(9.4 \%)$ \\
\hline other & $27(42.2 \%)$ \\
\hline Osteoporosis diagnosis based on, number (\%) & $17(26.6 \%)$ \\
\hline DXA (lumbar spine or femur) & $10(15.6 \%)$ \\
\hline - DXA of lumbar spine & $11(17.1 \%)$ \\
\hline - DXA of femur & $10(15.6 \%)$ \\
\hline osteoporotic fracture & $19(29.7 \%)$ \\
\hline Osteoporotic fractures, number (\%) & $12(18.9 \%)$ \\
\hline vertebrae & $2(3.1 \%)$ \\
\hline proximal femur & $5(7.8 \%)$ \\
\hline other low-energy fractures & $16(25 \%)$ \\
\hline Osteoporosis treatment, number (\%) & $14(21.9 \%)$ \\
\hline bisphosphonates & $2(3.1 \%)$ \\
\hline denosumab & \\
\hline GCs: Glucocorticoids. & \\
\hline
\end{tabular}

- Table 2 Correlations of parameters of bone density and architecture disturbances with age, BMI, and duration of GCs therapy.

\begin{tabular}{|c|c|c|c|}
\hline & Age & BMI & $\begin{array}{l}\text { Duration of } \\
\text { GCs therapy }\end{array}$ \\
\hline Lumbar BMD & $\begin{array}{l}r=-0.26 \\
p=0.0356\end{array}$ & ns & ns \\
\hline Lumbar T-score & ns & ns & ns \\
\hline Lumbar Z-score & ns & ns & ns \\
\hline Femoral BMD & $\begin{array}{l}r=-0.49 \\
p<0.0001\end{array}$ & ns & ns \\
\hline Femoral T-score & $\begin{array}{l}r=-0.39 \\
p=0.0039\end{array}$ & ns & ns \\
\hline Femoral Z-score & ns & ns & ns \\
\hline TBS & ns & $\begin{array}{l}r=-0.35 \\
p=0.0048\end{array}$ & ns \\
\hline T-score TBS & ns & $\begin{array}{l}r=-0.35 \\
p=0.0060\end{array}$ & ns \\
\hline
\end{tabular}

GCs: Glucocorticoids; ns: Correlation not significant. associated with significantly $(p=0.04)$ lower TBS T-scores compared to the male gender, but after adjusting for confounding factors lost its statistical significance.

The bone microarchitecture status and BMD status in patients with osteoporotic fractures are presented in $\triangleright$ Table 3 . There was no significant difference in the incidence of fractures in both groups - 9 patients with fractures in the group with osteoporosis diagnosed with BMD measurements and 10 in the group with disturbed bone microarchitecture according to TBS. No significant difference was also observed comparing patients with partially disturbed bone microarchitecture and osteopenia. The disturbed bone architecture was present in $30 \%$ of patients with fractures who did not meet the diagnostic criteria of osteoporosis based on DXA. In a group of patients with low-energy fractures, the univariate analysis showed significantly lower TBS $(p=0.02)$, TBS T-score $(p=0.012)$, femoral BMD ( $p=0.023)$, and older age $(p<0.0001)$. Additionally, the female gender was associated with a higher incidence of osteoporotic fractures $(p=0.013)$. However, after the multivariate analysis, only TBS $(p=0.033)$ and age $(p=0.002)$ remained significantly associated with the occurrence of osteoporotic fractures ( Fig. 1).

\section{Discussion}

GCs induce osteoporosis in several ways [1,2], including hormonal dysregulation (decreased production of gonadotrophins, estrogen, and testosterone; reduced growth hormone secretion; reduced gastrointestinal calcium absorption and renal calcium reabsorption) and disturbance of bone metabolism (leading to inhibition of the differentiation and replication of osteoblasts, induction of os-

- Table 3 The incidence (number, \%) of disturbed $(<1.23)$, intermediate $(\geq 1.23$ and $<1.31)$, or normal $(\geq 1.31)$ bone microarchitecture and BMD status.

\begin{tabular}{|c|c|c|c|}
\hline & $\begin{array}{l}\text { Disturbed } \\
\text { bone } \\
\text { microarchi- } \\
\text { tecture }\end{array}$ & $\begin{array}{l}\text { Intermediate } \\
\text { bone microar- } \\
\text { chitecture }\end{array}$ & $\begin{array}{l}\text { Normal } \\
\text { bone } \\
\text { microarchi- } \\
\text { tecture }\end{array}$ \\
\hline \multicolumn{4}{|l|}{ Osteoporosis } \\
\hline - lumbar DXA & $4(21.1 \%)$ & - & - \\
\hline - femoral DXA & $4(21.1 \%)$ & 1 (5.3\%) & $1(5.3 \%)$ \\
\hline - both & $7(36.8 \%)$ & $1(5.3 \%)$ & $1(5.3 \%)$ \\
\hline \multicolumn{4}{|l|}{ Osteopenia } \\
\hline - lumbar DXA & $5(26.3 \%)$ & $4(21.1 \%)$ & $2(10.5 \%)$ \\
\hline - femoral DXA & $5(26.3 \%)$ & $4(21.1 \%)$ & $2(10.5 \%)$ \\
\hline - both & $2(10.5 \%)$ & $5(26.3 \%)$ & $2(10.5 \%)$ \\
\hline \multicolumn{4}{|l|}{ Healthy } \\
\hline - lumbar DXA & $1(5.3 \%)$ & 2 (10.5\%) & $1(5.3 \%)$ \\
\hline - femoral DXA & $1(5.3 \%)$ & $1(5.3 \%)$ & - \\
\hline - both & $1(5.3 \%)$ & - & - \\
\hline
\end{tabular}



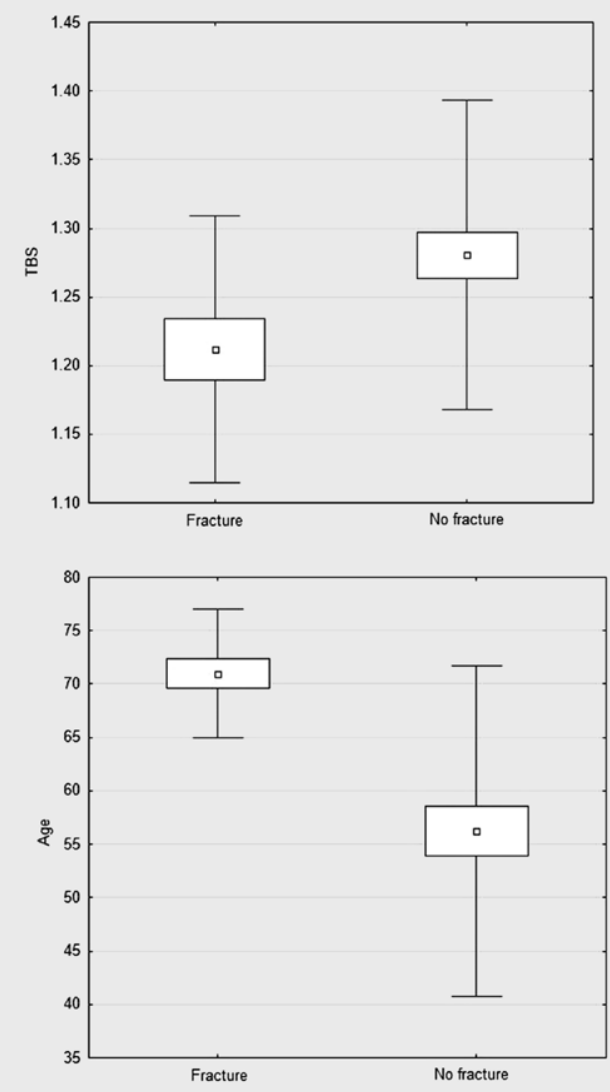

Fig. 1 Age and TBS in relation to the incidence of osteoporotic fractures, after adjusting for confounding factors.

teoblast and osteocytes apoptosis, and promoted osteoclast survival - resulting in increased bone resorption and reduction in bone formation). Alterations in bone metabolism may lead to an increased risk of fractures due to the effect on bone microarchitecture that occurs before the significant loss of BMD. The risk of fractures is further increased because of steroid-induced myopathy, which increases the risk of falls [1].

\section{Incidence of osteoporosis and fractures}

In our study, we diagnosed osteoporosis in $42.7 \%$ of patients, which is consistent with other reports, showing that osteoporosis can occur in up to $50 \%$ of patients with chronic GCs therapy $[1,2,16]$. Osteoporotic fractures occurred in $29.7 \%$ of our patients, which is also comparable to previous studies, reporting fractures in about $30-50 \%$ of patients with chronic GCs therapy $[1,2,16]$. The variability in the incidence of both osteoporosis and fractures can be explained by the differences in the characteristics of the patients studied (different age groups, menopausal status, doses, duration, and cause of GCs therapy) and the lack of a uniform diagnostic definition of GIO. Most of the osteoporotic fractures in our study were vertebral fractures (63\% of all fractures). This is in line with our knowledge that GCs mostly disrupt the structure of the trabecular bone that dominates in the vertebral bodies, and patients with $\mathrm{GIO}$ are at higher risk of vertebral fractures compared to hip fractures [1,2,4].

\section{Effect of glucocorticoids on bone microarchitecture}

The effects of chronic GCs therapy on bone microarchitecture (TBS) have been assessed in several previous studies. In the most recent study by Florenz et al. with 127 patients during chronic GC therapy disturbed bone microarchitecture was found in $52 \%$ of patients [16] - more frequently than in our study (40.6\% of all patients). They also found that disturbed microarchitecture occurred significantly more often than the BMD-based diagnosis of osteoporosis in patients with fractures (69 vs. $36 \%$ ). We did not find such a difference - in our study, the frequency of disturbed microarchitecture and osteoporosis diagnosed based on DXA BMD measurement in the group of patients with fractures was similar (52.6 vs. $47.4 \%$, respectively). As both studies used the same TBS thresholds, this difference may be caused by different rheumatological conditions treated with GCs in both studies - in our mainly rheumatoid arthritis and study by Florenz et al. vasculitis. Nonetheless, this discrepancy should be investigated further in future studies. Another issue that should be taken into account when assessing bone microarchitecture in patients treated with GCs is the negative correlation of TBS with BMI. A similar correlation was reported in the small study by Torgutalp et al. [20], suggesting that abnormalities of the bone tissue microarchitecture may be present more frequently in patients with higher BMI. Still, BMI was not associated with increased fracture occurrence in our study.

\section{TBS in fractures risk assessment}

In our study, in patients with low-energy fractures, we assessed not only osteoporosis diagnosed based on DXA BMD measurement and disturbed bone microarchitecture (TBS $<1.23$ ), but also intermediate states - osteopenia and partially disturbed bone microarchitecture ( $\triangleright$ Table 3 ). It is noteworthy that in patients with low-energy fractures only $15.8 \%$ had normal bone microarchitecture and $5.3 \%$ normal BMD. When all 3 bone parameters (TBS, femoral BMD, and lumbar BMD) were considered combined no patient with low-energy fracture had normal bone status. However, the most interesting part of our study is the analysis of risk factors for osteoporotic fractures, which suggests that TBS may be more sensitive than BMD for GCs-related fracture detection. In our study, both the univariate and multivariate analysis showed significantly lower TBS values in patients with the presence of low-energy fractures. Such a relationship for BMD-based parameters was not confirmed in the multivariate analysis. Similar results were found in a few previous studies. In the study by Leib et al., comparing 416 GCs treated patients with 1104 matched controls, there was a significant decrease of TBS values compared with the control group (while there was no change in BMD values). What's more a decrease in TBS values in GCs treated patients was associated with osteoporotic fractures, while there was no significant difference in BMD values between patients treated with GCs with and without osteoporotic fractures [12]. In the study by Choi et al., among 279 patients with rheumatoid arthritis and chronic GCs therapy, significantly lower values of TBS were found in patients with vertebral fractures compared to patients without vertebral fractures, with no difference in BMD values of lumbar spine between both groups [13]. These observations are further confirmed by studies reporting that TBS shows the better (higher) receiver operating characteristic areas under curves than lumbar spine BMD in patients with chronic GCs therapy with 
vertebral fractures [14-16]. FRAX adjusted with TBS also seems to be a better tool than ordinary FRAX for patients treated with GCs. Chuang et al. assessed the effect of GCs therapy on the values of TBS, BMD, FRAX, and FRAX adjusted with TBS during a 12-24 months interval pre-post study of 30 patients [21]. They found that while the treatment with GCs significantly reduced the TBS values and increased the risk of fractures according to FRAX adjusted with TBS, the GCs treatment did not significantly affect the values of $\mathrm{BMD}$ and FRAX in the multivariate analysis.

\section{Summary}

These results suggest that TBS with a properly selected cut-off point should be able to predict the occurrence of osteoporotic fractures in patients with chronic GCs treatment in a better way than BMD measured with DXA. In our study disturbed bone microarchitecture was diagnosed in $30 \%$ of patients with fractures and without a densitometric diagnosis of osteoporosis, confirming beyond doubt that TBS adds value to the classic DXA. The biggest limitation of our study is a relatively small sample size and the cross-sectional character of the study. Longitudinal observational studies that can demonstrate the usefulness of TBS in predicting osteoporotic fractures in patients with chronic GCs treatment would have the greatest clinical value. Up to date, only one study by Martineau et al. examined it using Manitoba BMD Registry [22]. They showed that lowering TBS by 1 SD in the group of patients with chronic GCs therapy increases major osteoporotic fractures HR to 1.15. Moreover, FRAX adjusted with TBS shows a modest but statistically significant improvement in predicting major osteoporotic fractures by $3.9 \%$. The other limitation of our study is the fact that we could not assess doses of GCs used, and in our analysis only the duration of GCs treatment was taken into account. The greatest strength of our study is the expansion of the available knowledge on the clinical utility of TBS in fracture risk assessment of patients with inflammatory rheumatic diseases treated with GCs, a topic that has been so far covered only by a few studies.

Our study proves that TBS reflects the influence of GCs therapy on bone quality better than BMD and provides an added value to classic DXA in identifying the group of patients particularly prone to fractures. However, prospective research is still needed to prove the usefulness of TBS in predicting fractures in patients treated with GCs.

\section{Conflict of Interest}

The authors declare that they have no conflict of interest.

\section{References}

[1] Lukert BP, Raisz LG. Glucocorticoid-induced osteoporosis: Pathogenesis and management. Ann Intern Med 1990; 112: 352-364

[2] De Nijs RNJ. Glucocorticoid-induced osteoporosis: A review on pathophysiology and treatment options. Minerva Med 2008; 99 : 23-43
[3] Cosman F, de Beur SJ, LeBoff MS et al. Clinician's Guide to Prevention and Treatment of Osteoporosis. Osteoporos Int 2014; 25: 2359-2381

[4] Van Staa TP, Leufkens HGM, Cooper C. The epidemiology of corticosteroid-induced osteoporosis: A meta-analysis. Osteoporos Int 2002; 13: 777-787

[5] Krohn K, Schwartz EN, Chung YS et al. Dual-energy X-ray Absorptiometry Monitoring with Trabecular Bone Score: 2019 ISCD Official Position. J Clin Densitom 2019; 22: 501-505

[6] Kanis JA, Cooper C, Rizzoli R et al. European guidance for the diagnosis and management of osteoporosis in postmenopausal women. Osteoporos Int 2019; 30: 3-44

[7] Ulivieri FM, Silva BC, Sardanelli F et al. Utility of the trabecular bone score (TBS) in secondary osteoporosis. Endocrine 2014; 47: 435-448

[8] Paggiosi MA, Peel NFA, Eastell R. The impact of glucocorticoid therapy on trabecular bone score in older women. Osteoporos Int 2015; 26: 1773-1780

[9] Leslie WD, Aubry-Rozier B, Lamy O et al. TBS (Trabecular Bone Score) and Diabetes-Related Fracture Risk. J Clin Endocrinol Metab 2013; 98 : 602-609

[10] Silva BC, Boutroy S, Zhang C et al. Trabecular Bone Score (TBS) - A Novel Method to Evaluate Bone Microarchitectural Texture in Patients With Primary Hyperparathyroidism. J Clin Endocrinol Metab 2013; 98 : 1963-1070

[11] Rampersad C, Whitlock RH, Leslie WD et al. Trabecular bone score in patients with chronic kidney disease. Osteoporos Int 2020; 31 : 1905-1912

[12] Leib ES, Winzenrieth R. Bone status in glucocorticoid-treated men and women. Osteoporos Int 2016; 27: 39-48

[13] Choi Y], Chung YS, Suh CH et al. Trabecular bone score as a supplementary tool for the discrimination of osteoporotic fractures in postmenopausal women with rheumatoid arthritis. Medicine (Baltimore) 2017; 96: e8661

[14] Kim HA, Lee HY, Jung JY et al. Trabecular Bone score is a useful parameter for the prediction of vertebral fractures in patients with polymyalgia rheumatica. J Clin Densitom 2020; 23: 373-380

[15] Kim D, Cho SK, Kim JY et al. Association between trabecular bone score and risk factors for fractures in Korean female patients with rheumatoid arthritis. Mod Rheumatol 2016; 26: 540-545

[16] Florez H, Hernández-Rodríguez J, Muxi A et al. Trabecular bone score improves fracture risk assessment in glucocorticoid-induced osteoporosis. Rheumatol (United Kingdom) 2020; 59: 1574-1580

[17] TBS report Hologic. Guidance and Notes (https://www.medimapsgroup.com/doc/follow-up-report/), Accessed 3 December 2020

[18] Assessment of fracture risk and its application to screening for postmenopausal osteoporosis. Report of a WHO Study Group. World Health Organ Tech Rep Ser 1994; 843: 1-129

[19] McCloskey EV, Odén A, Harvey NC et al. A Meta-Analysis of Trabecular Bone Score in Fracture Risk Prediction and Its Relationship to FRAX. J Bone Miner Res 2016; 31: 940-948

[20] Torgutalp ŞS, Babayeva N, Kara ÖS et al. Trabecular bone score of postmenopausal women is positively correlated with bone mineral density and negatively correlated with age and body mass index. Menopause 2019; 26: 1166-1170

[21] Chuang MH, Chuang TL, Koo M et al.Trabecular Bone Score Reflects Trabecular Microarchitecture Deterioration and Fragility Fracture in Female Adult Patients Receiving Glucocorticoid Therapy: A Pre-Post Controlled Study. Biomed Res Int 2017; 4210217

[22] Martineau P, Leslie WD, Johansson $\mathrm{H}$ et al. In which patients does lumbar spine trabecular bone score (TBS) have the largest effect? Bone 2018; 113: 161-168 